\title{
Real Time Online Sensing of Illicit Discharge of Wastewater into Lakes
}

\author{
K. K. Pranesh, M. Gayathri, S. Muthukeerthana, M. Elakkiya
}

\begin{abstract}
Monitoring the illicit discharge of effluent in water bodies is made easier with the present design. The water bodies are monitored using various sensors to know the illicit discharge of effluents. The water quality parameters that are detected by sensors are fed into NODE MCU micro controller for analysis and to compare the data and check with the predefined conditions. Smart sensor network has been designed in such a way that it predicts the overwhelming water quality parameters, for example, $\mathrm{pH}$, temperature, turbidity and electrical conductivity. The process of monitoring the effluents is carried out by the sensors. All the sensors are connected by sensor fusion technology through which they can compare their pre-set values and give us the accurate result on water quality status. It sends the message to the user about the standards of the effluent added to the water and also alert's the concern authority. All the process executed is saved in the Google drive of the device as a backup every day. This instrument can be placed in various locations of the lakes. The predefined values are as follows: the pH sensor will be maintained between 6-9, turbidity less than 10NTU. If the illicit discharge value exceeds these predefined parameters messages will be sent to the water maintenance department through message and the buzzer will make a sound. The GPS is enabled for anti-theft purpose.
\end{abstract}

Keywords: water quality, machine to machine mechanism, Google drive backup, buzzer, message to phone, discharge of effluents.

\section{INTRODUCTION}

In spite of the fact that the advances have been enticing in 21 st century, the centennial of contamination, enlistment warming, instability and exposed wellbeing variables wins the equivalent. Water contamination is the unrivalled issue before world till date, which is only the tainting of water bodies. Water contamination influences plants and animals living in these waterways. Likewise human wellbeing is influenced by contaminated water. It has been examined that water tainting is the fundamental wellspring of passing and ailments around the globe. The records show that more than 15,000 people kick the basin step by step far and wide. In India obvious 620 people kick the basin of water pollution related infection reliably. In many making countries, discoloured or water is being used for drinking with no authentic affirmed treatment. One purpose behind this occurrence is the numbness of open and association and the nonattendance of water quality checking system which makes real restorative issues. As water is the most huge factor for each living being, it is basic to verify it. Moreover water quality watching is one of the underlying advances required in the improvement and the officials of water resources. Subsequently in this paper we delineate the arrangement of steady online water sensors that screens the idea of water with the help of information identified by the sensors, without changing the possibility of the water to keep the water resource inside a standard depicted for private use and to have the alternative to take major exercises to restore the quality of the spoiled water body Using different sensors, this system can assemble various parameters of water, for instance, temperature, $\mathrm{pH}$, electrical conductivity, and turbidity.The fast improvement of remote sensor organize (WSN) innovation gives a novel way to deal with on-going information examination, control and execution .All the information's from different sensor are upheld up in the Google distributed storage which goes about as an open source. Consequently the unlawful release of influent is precisely distinguished by working with a predefined calculation. The principle target of this undertaking is to gauge water parameters, for example, $\mathrm{pH}$, temperature, turbidity, electrical conductivity, utilizing sensors wrapped by elastic pontoon which trap air and continue drifting in lakes.

\section{PROPOSED SYSTEM}

The principal point here is to develop a structure for incessant seeing of water quality using sensor frameworks with insignificant exertion and high area precision. $\mathrm{pH}$, temperature, electrical conductivity, turbidity are the parameters that are dismembered to find the illicit arrival of effluents in water bodies.

\section{METHODOLOGY}

The detailed methodology of this project is explained in figure 1.

Revised Manuscript Received on 14 August, 2019.

K.K.Pranesh, Student, Dept. of Civil Engineering, Sri Krishna College of Technology, Tamilnadu, India.(Email: 16tucv044@skct.edu.in)

M.Gayathri,Student, Dept. of Civil Engineering, Sri Krishna College of Technology,Tamilnadu, India.(Email: 16tucv012@skct.edu.in)

S.Muthukeerthana, Asst. Professor, Sri Krishna College of Technology, Tamilnadu, India.(Email: muthukeerthana.s@ skct.edu.in)

M.Elakkiya, Student, Dept. of I\&C Engineering, Sri Krishna College of Technology, Tamilnadu, India.(Email: 16tuic016@ skct.edu.in) 


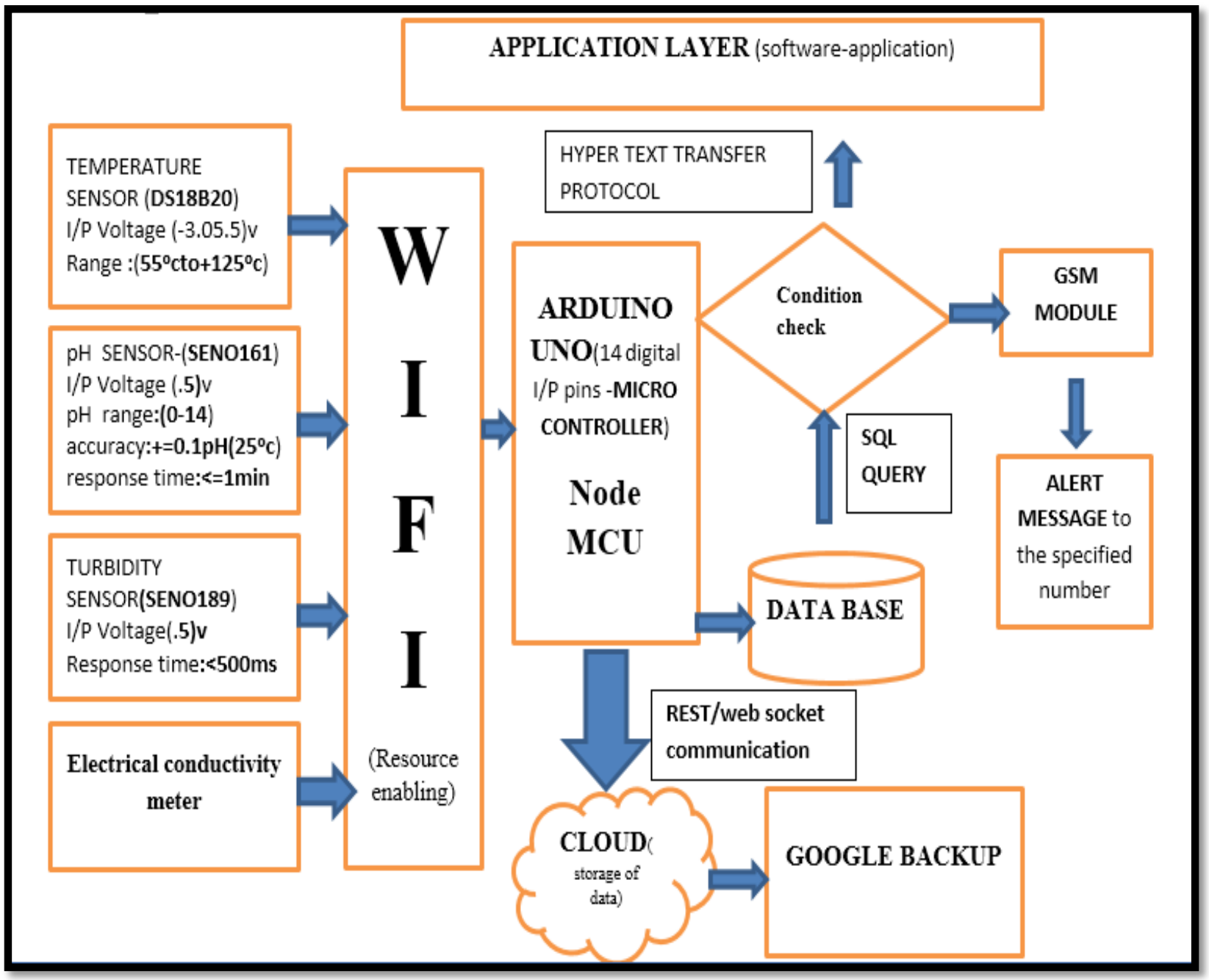

Figure 1 Methodology of real time online sensing of illicit discharge of waste water into lakes

Node MCU is used as the micro controller with full TCP/IP stack and build-in Wi-Fi module. Different sensors that measures corresponding parameters are connected as a single unit using Sensor Fusion technology due to which all the parameters are relatively compared and produce results based on predefined algorithms. The sensors are vertically aligned and wrapped with rubber raft so that it can trap the atmospheric air and keep floating. For extreme accuracy all the corners and the midpoint of the lake are continuously monitored using sensors wrapped rubber rafts. The results are pushed to the cloud by using Blynk server which acts as an application layer using this layer the results can be shared (both by SMS and mail) to concern authorities. All the data's from various sensors are successfully saved in Google driver as a backup. GPS module is also used as an anti-theft purpose.

\section{RESULTS AND DISCUSSION}

The data are collected from various sensor nodes and sent through resource enabling Wi-Fi process, in order to simulate and analyse quality parameters, ARDUINOUNO(14 digital I/P pins -microcontroller)and Node MCUacts as a micro controller. Depending on the data's collected by the sensor node, data analytics is done using PYTHON TOOL in the local database. Data analytics is done to predict the nearest future value so that precautions can be made prior. The buzzer will be fixed, subsequently when there is a deviation in the parameters from the pre-set qualities, buzzer gets triggered and simultaneously an alert message will be send to the concern authority. The rubber raft material mush is periodically changed with an interval of six months. The GSM module has been fixed for the message alert, GPS system is also incorporated for anti-theft purpose. Thus, when water quality recognized does not coordinate the present models, fundamental moves can be made by the authorities.

The sensors which are used for detecting the water quality are, 1. Temperature sensor (ds18b20), I/p voltage (-3.05.5) $\mathrm{v}$, range :( $55^{\circ} \mathrm{c}$ to $\left.+125^{\circ} \mathrm{c}\right)$. 2. pH sensor-(seno161), i/p voltage $(0.5) \mathrm{v}, \mathrm{pH}$ range $:(0-14)$, accuracy: $+=0.1 \mathrm{ph}\left(25^{\circ} \mathrm{c}\right)$, response time: less than or equal to $1 \mathrm{~min}$. 3 . Turbidity sensor (seno189), i/p voltage (.5) v, response time: less than $500 \mathrm{~ms}$. 4. Electrical conductivity meter. The result displayed is shown in Figure 2. 


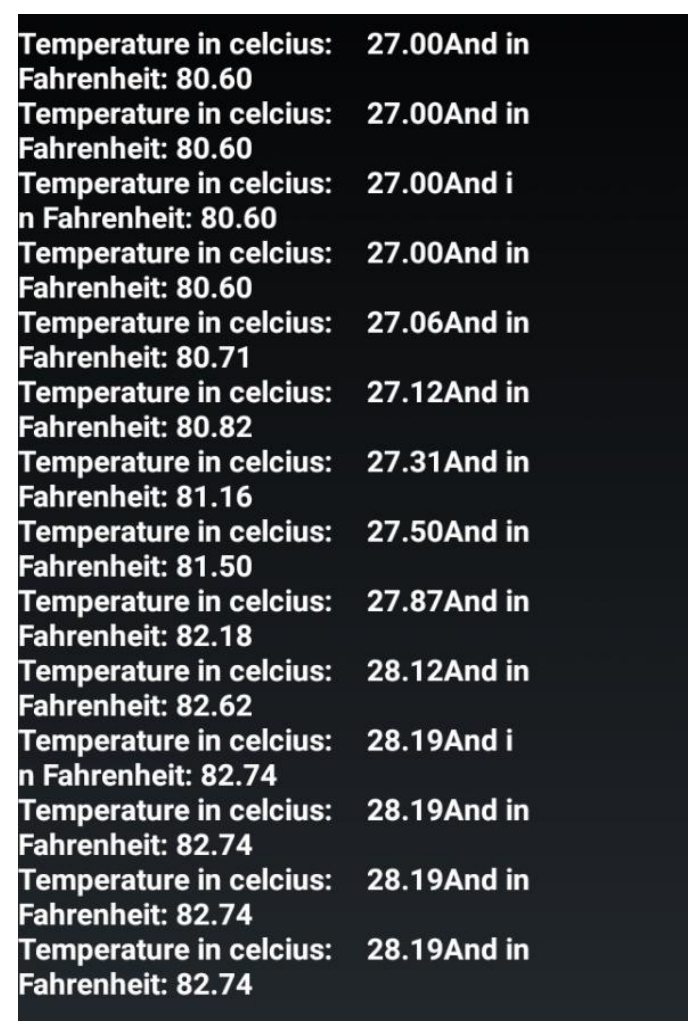

Figure 2 Screen shot of result from PC

\section{ADVANTAGES}

The main advantages of this project are that the Pollution control board office can check the problem when they receive the message at any time and at any place, the public will be benefited and come to know about the illicit discharge by the buzzer alarm sound, it is an error free system and it is $24 * 7$ system (continuous), it can also be used in other applications like aquaculture. This system produces an accurate result as it works on algorithm bases and it is completely inter operable in nature. It consumes less power and convenient to work with batteries.

\section{CONCLUSION}

This project blooms to find a smart solution for a big existing problem by online monitoring of water bodies which is the need for hour. Real scale studies will be implemented in future. This framework gives a caution to the remote client, when there is a deviation of water quality parameters from the pre-characterized set of standard qualities. Online monitoring of water bodies helps the officials to take stringent measures against the polluter.

\section{REFERENCES}

1. Jiang, P. Survey on Key Technology of WSN-Based Wetland Water Quality Remote Real-Time Monitoring System. Chin. J. Sens. Actuat. 2007, 20, 183-186.

2. Akyildiz, L.F.; Su, W.; Sankarasubramaniam, Y.; Cayirci, E. Wireless Sensor Networks: A Survey. Comput. Netw. 2002, 38, 393-422.

3. Mills, D.L. Internet Time Synchronization: The Network Time Protocol. IEEE Trans. Commun. 1991, 39, 14821493.

4. Huang X, Yi J, Chen S, Zhu X.Sensors (Basel). 2015 Nov 19;15(11):29273-96. 10.3390/s151129273.PMID:26610496.
5. International Journal on Recent and Innovation Trends in Computing and Communication ISSN: 2321-8169 Volume: 5 Issue: $2302-305$

6. Walker, W.R., Hrezo, M.S., and Haley, C.J., 1991, Management of Water Resources for Drought Conditions, in Paulson, R.W., Chase, E.B., Roberts, R.S., and Moody, D.W., Compilers, National Water Summary 1988-89--Hydrologic Events and Floods and Droughts: U.S. Geological Survey Water-Supply Paper 2375, p 147-156.

7. Real Time Water Quality Monitoring System ISSN(Online): 2320-9801 ISSN (Print): 2320-9798 International Journal of Innovative Research in Computer and Communication Engineering (An ISO 3297: 2007 Certified Organization) Vol. 3, Issue 6, June 2015 Copyright to IJIRCCE DOI: 10.15680/ijircce.2015.03060165064.

8. Smart Water Monitoring System Using Wireless Sensor Network at Home/Office 1Ms T.Deepiga, 2Ms A.Sivasankari. International Research Journal of Engineering and Technology (IRJET) e-ISSN: 23950056 Volume: 02 Issue: 04 | July-2015 www.irjet.net pISSN: 2395-0072 (C) 2015, IRJET.NET- All Rights Reserved Page 1305.

9. Bai, H.X., Chen, X., Guan, X.H.: Preserving Coverage for Wireless Sensor Networks of Nodes with Various Sensing Ranges. In: ICNSC 2006. Proceedings of the 2006 IEEE International Conference, pp. 54-59. IEEE Press, Los Alamitos (2006).

10. Yadav, Poonam\&Yadav, Nagesh\&Varma, Shirshu. (2007). Cluster Based Hierarchical Wireless Sensor Networks (CHWSN) and time synchronization in CHWSN. 1149 - 1154. 10.1109/ISCIT.2007.4392190.

11. Harrop P (2012) Wireless sensor networks and the new Internet of things. Energy Harvest J. Available at http://www.energyharvestingjournal.com.

12. Geetha, S \&Gouthami, Shiramshetty. (2017). Internet of things enabled real time water quality monitoring system. Smart Water. 2. 1. 10.1186/s40713-017-0005-y.

13. Shakkottai, S., Srikant, R., Shroff, N.: Unreliable Sensor Grids: Coverage, Connectivity and Diameter. In: Bauer, F. (ed.) Proc. of the IEEE Infocom, pp. 1073-1083. IEEE Press, San Francisco (2003).

14. Naik P, Sivalingam K (2004) A survey of MAC protocols for sensor networks. In: Wireless sensor networks, pp 93-107. 